\title{
A Study on the Pulmonary Manifestations of Rheumatoid Arthritis from a North Indian Town
}

\author{
Nazish Fatima ${ }^{1 *}$, Mohammed Shameem ${ }^{2}$, Abida Malik ${ }^{1}$, Parvez Anwar Khan ${ }^{1}$, \\ Fatima Shujatullah $^{1}$, Sohail Ahmed ${ }^{3}$, Nabeela ${ }^{1}$ \\ ${ }^{1}$ Department of Microbiology Jawaharlal Nehru Medical College, Aligarh Muslim University, Aligarh, India \\ ${ }^{2}$ Department of Tuberculosis and Chest Diseases Jawaharlal Nehru Medical College, \\ Aligarh Muslim University, Aligarh, India \\ ${ }^{3}$ Department of Orthopedics Jawaharlal Nehru Medical College, Aligarh Muslim University, Aligarh, India \\ Email: *nazsham28@yahoo.co.in
}

Received January 21, 2013; revised February 23, 2013; accepted March 3, 2013

Copyright (c) 2013 Nazish Fatima et al. This is an open access article distributed under the Creative Commons Attribution License, which permits unrestricted use, distribution, and reproduction in any medium, provided the original work is properly cited.

\begin{abstract}
Rheumatoid arthritis (RA) is a chronic systemic disease of unknown etiology characterized by articular involvement, extra-articular involvement, and the presence of serum rheumatoid factor. Pulmonary involvement in RA is a common extra-articular manifestation of rheumatoid arthritis (RA) that confers significant morbidity and mortality. We undertook this study to determine the prevalence and spectrum of pulmonary abnormalities in patients with rheumatoid arthritis (RA) from a North Indian town. 62 patients who met the American College of Rheumatology (formerly the American Rheumatism Association) 1987 classification criteria for RA were subjected to clinical examination of chest, X-Ray-chest (CXR), pulmonary function tests (PFT) and high resonance computed tomography (HRCT). 40.3\% patients had some pulmonary symptoms with exertional dyspnoea in $21 \%$, cough with expectoration in $17.7 \%$, fine respiratory rales in $11.3 \%$, patients X-ray chest bilateral lower zone haziness in $16 \%$ and prominent pulmonary vasculature in 3.2\%. 43\% had abnormal PFT-restrictive pattern in 29\%, obstructive pattern 8\% and mixed pattern in 6.4\%. HRCT revealed abnormal findings in 33.8\% commonest being ground glass pattern in both lower lobes 19.3\%, sub pleural reticulations in $9.6 \%$, pleural thickening in 3.2\% and pulmonary vascular prominence in $1.6 \%$. To provide optimal treatment, physicians must always consider the possibility of associated pulmonary manifestations when patients with RA are evaluated.
\end{abstract}

Keywords: Rheumatoid Arthritis; Pulmonary Manifestations

\section{Introduction}

Rheumatoid arthritis (RA) is a chronic systemic disease of unknown etiology characterized by articular involvement, extra-articular involvement and the presence of serum rheumatoid factor. In RA, there is a symmetric and persistent inflammation of the synovial tissue, usually involving the peripheral joints. Patients with RA who have high titers of rheumatoid factor, i.e., autoantibodies to the Fc component of immunoglobulin $G$ are most likely to have extra-articular manifestations of their disease, including rheumatoid nodules, rheumatoid vasculitis, and pleuropulmonary, neurologic, digestive, cardiovascular, cutaneous, hematologic and ocular complications [1]. Pulmonary involvement in RA is a common extra-articular manifestation of RA [2] that confers sig-

${ }^{*}$ Corresponding author. nificant morbidity and mortality [3].

Given the impact of lung disease on morbidity and mortality in RA, screening of asymptomatic RA patients for pulmonary involvement has been recommended by some experts [4-6]. We undertook this study to determine the prevalence and spectrum of pulmonary abnormalities in patients with RA from a North Indian town.

\section{Material \& Method}

5661 patients presenting with complaints of arthritis at the orthopedics OPD, JN Medical College between Jan 2007-Dec 2011 were tested for serum rheumatoid factor by latex agglutination test. Of these, 62 patients who met the American College of Rheumatology (formerly the American Rheumatism Association) 1987 classification criteria for RA [7] (Table 1) were included in the study. The patients who met the American College of Rheuma- 
tology Criteria were further referred to Respiratory Disease centre for pulmonary evaluation. They were subjected to clinical examination of chest, X-Ray-chest (CXR), pulmonary function tests (PFT) and high resonance computed tomography (HRCT).

Signs and symptoms were recorded from each patient based on a set of questionnaires focusing on cough, sputum production, wheezing and dyspnoea. History of smoking was also recorded. This questionnaire meets American Thoracic Society criteria for epidemiologic surveys in chronic respiratory diseases [8] and is considered reproducible, valid and free of bias [9].

PFTs were performed on RA patients using a standard protocol. Vital capacity (VC), forced vital capacity (FVC), residual volume (RV), total lung capacity (TLC), forced expiratory volume in the first second $\left(\mathrm{FEV}_{1}\right)$, $\mathrm{FEV}_{1} / \mathrm{FVC}$ ratio (a sensitive index of overall airway obstruction), and forced expiratory flow from $25 \%$ to $75 \%$ of $\mathrm{VC}\left(\mathrm{FEF}_{25-75}\right.$, a specific index of small airway function) were measured by spirometry.

HRCT findings for lung parenchymal abnormalities that included airspace consolidation, ground-glass opacity (GGO), reticulation, honeycombing, nodules etc. were assessed.

\section{Results}

Of the 5661 patients who presented with history of arthritis, 62 (1.1\%) patients met the ACR criteria 1987 of RA, of which, 47 were females and 15 were males (Female: male ratio 3:1). 25(40.3\%) patients had some pulmonary symptoms with exertional dyspnoea in 13 (21\%), cough with expectoration in 11 (17.7\%), fine respiratory rales in 7 (11.3\%) patients. 12 (19.3\%) gave history of smoking (Table 2). X-ray chest revealed an abnormality in 15 (24\%) cases, commonest pattern being bilateral-lower zone haziness in 10 (16\%) and prominent pulmo-

Table 1. American College of Rheumatology revised criteria for diagnosing rheumatoid arthritis.

\begin{tabular}{|c|c|}
\hline \multirow{2}{*}{$\begin{array}{l}\text { Guidelines for } \\
\text { classification }\end{array}$} & $\begin{array}{l}\text { At least } 4 \text { of } 7 \text { criteria are required to classify a } \\
\text { patient as having rheumatoid arthritis. }\end{array}$ \\
\hline & $\begin{array}{l}\text { Patients with } 2 \text { or more clinical diagnoses are } \\
\text { not excluded. }\end{array}$ \\
\hline \multirow{7}{*}{ Criteria } & Morning stiffness* \\
\hline & Arthritis of 3 or more joints* \\
\hline & Arthritis of hand joints* \\
\hline & Symmetric arthritis ${ }^{*}$ \\
\hline & Rheumatoid nodules \\
\hline & Serum rheumatoid factor \\
\hline & Radiographic changes \\
\hline
\end{tabular}

*Must be present for at least 6 weeks. nary vasculature in 2 (3.2\%) (Table 3). 27 patients (43\%) had abnormal PFT-restrictive pattern in 18 (29\%), obstructive pattern 5 (8\%) and mixed pattern in 4 (6.4\%). HRCT revealed abnormal findings in 21 (33.8\%) commonest being ground glass pattern in both lower lobes 12 (19.3\%), sub pleural reticulations in 6 (9.6\%), pleural thickening in 2 (3.2\%) and pulmonary vascular prominence in 1 (1.6\%) (Table 4).

\section{Discussion}

The prevalence of RA is around 1\% worldwide. The information about prevalence of RA in India is scarce [10]. In a study on the prevalence of rheumatoid arthritis in the adult Indian population by Malviya et al., a prevalence rate of $0.75 \%$ was found [11]. The Indian prevalence rate (0.9\%) almost equals the world prevalence rate [12]. Our study showed a slightly higher prevalence rate (1.1\%). This could be because the patients included were those presenting with history of arthritis.

RA predominantly occurs in females with women suffering 3 - 5 times more than males [12]. This study also revealed a much higher incidence rate (Female:male ratio 3:1) among females. The variation in the level of sex hormones of females (oestrogen and progesterone,

Table 2. Patient characteristic according to the presence of any pulmonary function abnormalities.

\begin{tabular}{cccc}
\hline Any symptoms & $(\mathrm{N})$ PFT $\mathrm{n}=35$ & $\mathrm{Ab}(\mathrm{N}) \mathrm{PFT} \mathrm{n}=27$ & Total \\
\hline Cough & $10(36 \%)$ & $15(58 \%)$ & 25 \\
Sputum & $4(11 \%)$ & $7(25.9 \%)$ & 11 \\
Wheezing & $2(5.7 \%)$ & $5(18.5 \%)$ & 7 \\
Dyspnoea & $4(11.4 \%)$ & $9(33.3 \%)$ & 13 \\
Smoking & $2(5.7 \%)$ & $10(37 \%)$ & 12 \\
\hline
\end{tabular}

Table 3. X-Ray chest of RA patient.

\begin{tabular}{ccc}
\hline & Patient No. & Percentage (\%) \\
\hline Normal & 47 & $76 \%$ \\
B/L Lower zone haziness & 10 & $16 \%$ \\
Prominent Pulmonary vasculature & 2 & $3.2 \%$ \\
\hline
\end{tabular}

Table 4. HRCT finding in RA patient.

\begin{tabular}{ccc}
\hline & Patient No. & Percentage (\%) \\
\hline Normal & 41 & $79 \%$ \\
Ground glass pat (both lower lobes) & 12 & $19.3 \%$ \\
Sub pelural reticulations & 6 & $9.6 \%$ \\
Pleural thickening & 2 & $3.2 \%$ \\
Pulmonary vascular prominence & 1 & $1.6 \%$ \\
\hline
\end{tabular}


which regulate the inflammatory process) is the main cause of development of RA among them [13].

Of the RA cases studied for pulmonary involvement, nearly one-fourth (21\%) gave history of dyspnoea and a productive cough. These symptoms are a common presentation in diffuse interstitial fibrosis of the lungs. [1]. Dyspnoea may also point towards pleural effusion, pulmonary vasculitis or to a chest infection [1], while wheeze seen in $11 \%$ patients may indicate an obstructive pulmonary disease.

The predominant presentation on X-ray Chest was a bilateral lower zone diffuse shadow (16\%) predominantly, reticular (Figure 1) and nodular pattern and prominent pulmonary vasculature. These findings are suggestive of an underlying interstitial lung disease. Pleural effusion was the second common finding on CxR which is the most common asymptomatic pulmonary complication of RA. Also, prominent pulmonary vasculature could be due to pulmonary vasculitis which is a less common pulmonary manifestation of RA.

Majority of RA patients had a restrictive pattern on pulmonary function testing which again highlights the presence of lung fibrosis and an underlying interstitial lung disease [14]. 8\% of our RA cases had obstructive pattern with airflow obstruction that was most marked when respiratory volumes were low and trapping of air occurred. It was found that patients with history of smoking predominantly revealed an obstructive pattern on PFT. The mean forced expiratory volume in 1 second and forced vital capacity are reduced. The combination of tobacco smoking and RA is associated with a much higher prevalence of obstructive pulmonary disease [15].

On HRCT, majority of RA patients (19\%) presented with ground-glass opacity (GGO) which may be the result of air space disease (filling of the alveoli) or interstitial lung disease (ILD) (i.e. fibrosis). Second common

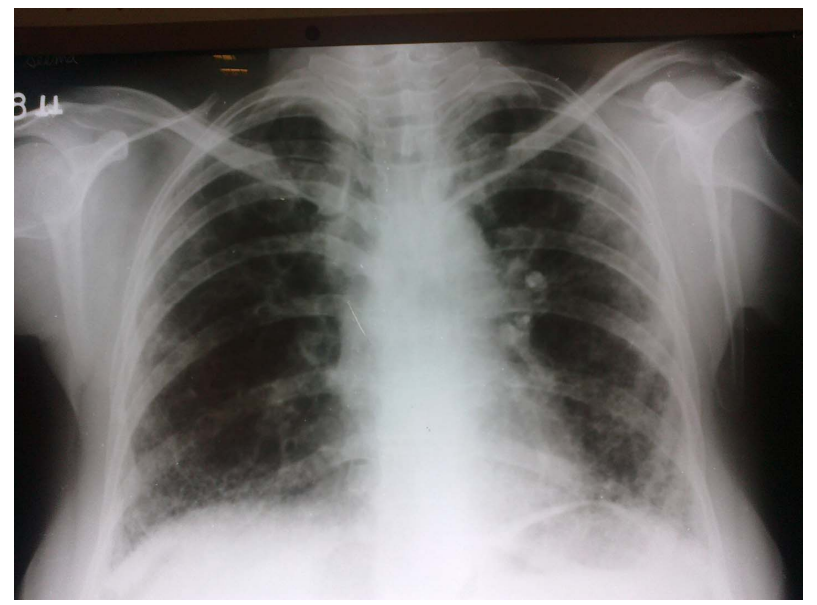

Figure 1. Chest X Ray (PA-View) showing bilateral basal reticular shadows. finding on HRCT was the presence of reticular pattern which again indicates fibrosis (Figure 2).

Thus, the observations in our study are in agreement with other studies that ILD is the commonest pulmonary manifestation in RA patients [14,16,17]. The interstitial lung diseases are comprised of a group of pulmonary disorders characterized clinically by diffuse infiltrates on the chest radiograph and histologically by distortion of the gas exchanging portion of the lung. The physiologic correlates are restriction of lung volumes and impaired oxygenation. The lungs of patients with interstitial disease show varying degrees of fibrosis and inflammation. Fibrosis is characterized by an increased amount and abnormal structure of the connective tissue while inflammation is characterized by excessive inflammatory cells. Inflammation is a characteristic cellular and molecular response to injury, noxious exposure, or infectious agents. Fibrosis, or scarring, can be part of the healing response to injury, but fibrosis as a disease process occurs when restoration to normal tissue does not occur. Many of the cells and chemical mediators involved in inflammation are also involved in fibrosis. Fibrosis occurs when the laying down of collagen and other connective tissue does not stop and allow a return to the normal structure. Probably a recurrent immune stimulation occurs with the autoimmune disease such as in rheumatoid arthritis which causes lung injury.

The most sensitive method for detecting ILD is HRCT of chest but this technique is expensive especially for resource poor setting like India. Also, it is associated with significant radiation exposure [18], limiting its suitability for screening of asymptomatic individuals. PFTs are low risk-diagnostic modality that have been proved valuable in early detection of RA-associated lung disease. Nevertheless the cost of screening of all asymptomatic RA patients with PFTs makes this approach untenable. Clinicians may therefore rely on development of overt respiratory symptoms and/or physical findings in RA patients

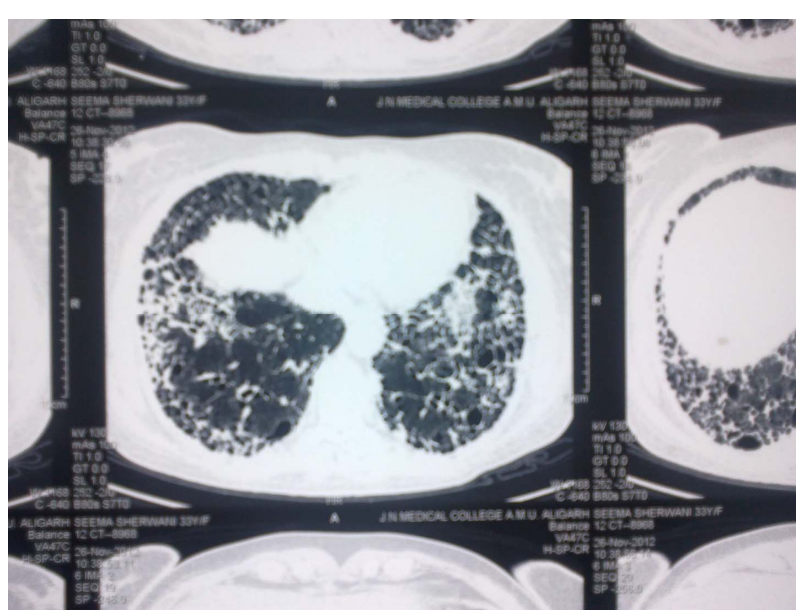

Figure 2. Honey comb appearance of lungs on HRCT. 
as trigger for evaluation of lung disease.

\section{Conclusion}

It is suggested that respiratory symptoms and PFT abnormalities can be used as predictors of lung disease. To provide optimal treatment, physicians must always consider the possibility of associated pulmonary manifestations when patients with RA are evaluated. It is concluded that early identification and timely therapeutic intervention may alter the prognosis of pulmonary fibrosis in patients of Rheumatoid arthritis [19,20].

\section{REFERENCES}

[1] K. Chanin, F. Vallejo-Manzur, G. L. Sternbach, et al., "Pulmonary Manifestations of Rheumatoid Arthritis," Hospital Physician, 2001, pp. 23-28.

[2] C. Turesson and L. T. Jacobsson, "Epidemiology of ExtraArticular Manifestations in Rheumatoid Arthritis," Scandinavian Journal of Rheumatology, Vol. 33, No. 2, 2004, pp. 65-72. doi:10.1080/03009740310004621

[3] C. Turesson, L. Jacobsson and U. Bergstrom, "ExtraArticular Rheumatoid Arthritis: Prevalence and Mortality,” Rheumatology (Oxford), Vol. 38, No. 7, 1999, pp. 668-674. doi:10.1093/rheumatology/38.7.668

[4] B. R. Gochuico, N. A. Avila, C. K. Chow, L. J. Novero, H. P. Wu, P. Ren, S. D. MacDonald, W. D. Travis, M. P. Stylianou and I. O. Rosas, "Progressive Preclinical Interstitial Lung Disease in Rheumatoid Arthritis,” Archives of Internal Medicine, Vol. 168, No. 2, 2008, pp. 159-166. doi:10.1001/archinternmed.2007.59

[5] V. Saravanan and C. A. Kelly, "Reducing the Risk of Methotrexate Pneumonitis in Rheumatoid Arthritis," Rheumatology (Oxford), Vol. 43, No. 2, 2004, pp. 143-147. doi:10.1093/rheumatology/keg466

[6] C. Kelly and V. Saravanan, "Treatment Strategies for a Rheumatoid Arthritis Patient with Interstitial Lung Disease," Expert Opinion on Pharmacotherapy, Vol. 9, No. 18, 2008, pp. 3221-3230. doi:10.1517/14656560802591430

[7] F. C. Arnett, S. M. Edworthy, D. A. Bloch, D. J. McShane, J. F. Fries, N. S. Cooper, L. A. Healey, S. R. Kaplan, M. H. Liang, H. S. Luthra, T. A. Medsger and R. L. Hunder, "The American Rheumatism Association 1987 Revised Criteria for the Classification of Rheumatoid Arthritis,” Arthritis Rheum, Vol. 31, No. 3, 1988, pp. 315324. doi:10.1002/art.1780310302

[8] C. LeMaistre, "A Statement by the Committee on Standards for Epidemiology Surveys on Chronic Respiratory Disease of the American Thoracic Society," National Tuberculosis and Respiratory Disease Association, New
York, 1969, p. 32.

[9] "Recommended Respiratory Disease Questionnaires for Use with Adults and Children in Epidemiological Research,” 1978, pp. 7-53.

http://www.thoracic.org/statements/resources/archive/rrdq uacer.pdf

[10] E. Akhtar, S. Bilal, et al., "Prevelence of Arthritis in India and Pakistan: A Review," Rheumatology International, Vol. 31, No. 7, 2011, pp. 849-855. doi:10.1007/s00296-011-1820-3

[11] A. N. Malviya, S. K. Kapoor, et al., "Prevelence of Rheumatoid Arthritis in the Adult Indian Population,” Rheumatology International, Vol. 13, No. 4, 1993, pp. 13-14.

[12] P. Milind and K. Sushila, "How to Live with Rheumatoid Arthritis," International Research Journal of Pharmacy, Vol. 3, No. 3, 2012, pp. 115-121.

[13] L. M. Pamell, C. L. Galligon and C. N. Fish, "Sex Affects Immunity Autoimmune,” 2012.

[14] F. R Lake, "Interstitial Lung Disease in Rheumatoid Artritis,” 2012, pp. 1-4. http://www.uptodate.com/contents/interstitial-lung-diseas e-in-rheumatoid-arthritis

[15] R. L. Collin, R. A. Turner, A. M. Johnson, et al., "Obstructive Pulmonary Disease in Rheumatoid Arthritis," Arthritis Rheum, Vol. 19, No. 3, 1976, pp. 623-628. doi:10.1002/art.1780190316

[16] E. Gabbay, R. Tarala, R. Will, et al., "Interstitial Lung Disease in Recent Onset Rheumatoid Arthritis," American Journal of Respiratory and Critical Care Medicine, Vol. 156, No. 2, 1997, pp. 528-535. doi:10.1164/ajrccm.156.2.9609016

[17] J. K. Dawson, H. E. Fewins, J. Desmond, et al., "Fibrosing Alveolitis in Patient with Rheumatoid Arthritis as Assessed by High Resolution Computed Tomography, Chest Radiography and Pulmonary Function Tests," Thorax, Vol. 56, No. 8, 2001, pp. 622-627. doi:10.1136/thorax.56.8.622

[18] E. J. Hall and D. J. Brenner, "Cancer Risks from Diagnostic Radiology,” British Journal of Radiology, Vol. 81, No. 965, 2008, pp. 362-378. doi:10.1259/bjr/01948454

[19] J. W. Card, W. J. Racz, J. F. Brien, S. B. Margolin and T. E. Massey, "Differential Effects of Pirfenidone on Acute Pulmonary Injury and Ensuing Fibrosis in the Hamster Model of Amiodarone-Induced Pulmonary Toxicity,” Toxicological Sciences, Vol. 75, No. 1, 2003, pp. 169-180. doi:10.1093/toxsci/kfg167

[20] W. A. Gahl, M. Brantly, J. Troendle, N. A. Avila, A. Padua, C. Montalvo, H. Cardona, K. A. Calis and B. Gochuico, "Effect of Pirfenidone on the Pulmonary Fibrosis of Hermansky-Pudlaksyndrome,” Molecular Genetics and Metabolism, Vol. 76, No. 3, 2002, pp. 234-242. doi:10.1016/S1096-7192(02)00044-6 